\title{
Introduction : La désindustrialisation, objet géographique
}

Introduction: Deindustrialization, a geographical phenomenon

Entindustrialisierung, geographisches Objekt

\section{François Bost}

\section{(2) OpenEdition}

Journals

Édition électronique

URL : http://journals.openedition.org/rge/6332

ISSN : 2108-6478

Éditeur

Association des géographes de l'Est

Référence électronique

François Bost, «Introduction : La désindustrialisation, objet géographique », Revue Géographique de l'Est [En ligne], vol.57 / 1-2 | 2017, mis en ligne le 15 novembre 2017, consulté le 08 septembre 2020. URL : http://journals.openedition.org/rge/6332

Ce document a été généré automatiquement le 8 septembre 2020

Tous droits réservés 


\title{
Introduction : La désindustrialisation, objet géographique
}

\author{
Introduction: Deindustrialization, a geographical phenomenon \\ Entindustrialisierung, geographisches Objekt
}

François Bost

\section{Introduction}

1 Un certain consensus existe entre les différentes approches économiques européennes pour faire de la baisse de l'emploi industriel un indicateur essentiel de la désindustrialisation. Le dictionnaire Larousse propose par exemple de définir cette dernière comme la " réduction du nombre d'emplois dans le secteur industriel d'un pays, de même que celle du secteur de l'industrie par rapport aux autres secteurs d'activité». Sous sa forme la plus brutale et la plus spectaculaire, la désindustrialisation se traduit par la fermeture d'usines dans les territoires et les bassins d'emplois. Mais son action s'exerce aussi de manière plus insidieuse et moins visible, donc moins médiatique : suppression de certaines lignes de fabrication ou d'assemblage (les usines restent en place, mais leur production est diminuée); abandon de certaines étapes du processus de production dans le pays d'origine et, en compensation, recours à un approvisionnement depuis l'étranger pour des composants et des sous-produits.

2 Par effectifs entiers, ou par grappes de quelques dizaines ou de quelques postes seulement, la désindustrialisation se traduit à chaque fois par la suppression - le plus souvent définitive - de nombreux emplois. Lorsqu'elle ne correspond pas à une augmentation de la productivité du travail (notamment sous l'effet de la robotisation des tâches), la désindustrialisation s'accompagne aussi par le transfert ou la délocalisation d'activités industrielles vers des pays plus compétitifs, notamment ceux où la main-d'œuvre est moins couteuse (Bost, 2014). À des degrés divers, tous les pays développés ont été touchés à partir des années 1970 par la désindustrialisation de leurs 
systèmes productifs. Accepté d'abord comme un phénomène inéluctable (celui du passage de la société industrielle à celui de la société post-industrielle), ce processus a pris un tour résolument inquiétant à la faveur de la crise économique mondiale apparue en 2007-2008 et qui a fait office de révélateur de son ampleur.

Présentée désormais comme l'une des conséquences majeures de la mondialisation de l'économie, dont elle serait l'une de ses faces sombres pour les pays développés, la désindustrialisation fait depuis plusieurs années les choux gras des éditorialistes de la presse européenne, des observateurs et autres "déclinologues», pour qui ce phénomène participerait au "déclin » des pays développés. Ces derniers seraient en quelque sorte entrés dans une nouvelle catégorie, peu enviable, celle des " pays en voie de désindustrialisation ».

4 Mais qu'en est-il vraiment ? Quelle est l'ampleur réelle de ce phénomène et comment le mesure-t-on le plus efficacement? Comment se traduit-il en Europe, région du monde qui a vu précisément débuter la Révolution industrielle? À quelles conditions une réindustrialisation de ces pays et des territoires anciennement industrialisés est-elle envisageable?

5 Ce numéro spécial de la Revue Géographique de l'Est entend analyser ce phénomène de la désindustrialisation à partir de quelques exemples de pays européens choisis pour leur représentativité (France, Allemagne, Pologne, Espagne), ou de cas d'école régionaux (Lorraine) ou urbain (Łódź en Pologne).

\section{L'Europe, un continent particulièrement concerné}

Établi à partir de la base de données AMECO de la Commission européenne, le tableau ci-dessous dresse pour plusieurs grands pays particulièrement représentatifs un état des lieux saisissant de l'ampleur du phénomène de désindustrialisation durant la période 1991-2010.

7 Berceau de la Révolution industrielle, l'Europe apparait notamment comme le continent le plus frappé par la baisse de l'emploi industriel, notamment dans sa composante manufacturière, et ce depuis le milieu des années 1970. Il est vrai que le grand nombre de pays concernés dans cette région du monde renforce encore l'impact de ce constat.

8 Sur la période étudiée, la France et l'Allemagne affichent ainsi une baisse de l'emploi manufacturier assez proche en valeur relative (respectivement $-30,7 \%$ et $-28,7 \%$ ), mais bien différente en valeur absolue (avec respectivement 900000 et 2 millions d'emplois), ce qui montre bien que même un pays comme l'Allemagne, dont l'image reste celle d'un pays encore très fortement industrialisé, n'échappe pas à cette lame de fond.

9 L'effondrement est encore plus important au Royaume-Uni (- $41 \%$ ) et au Japon (- $32 \%)$, ce dernier apparaissant même comme le plus grand perdant en valeur absolue en matière de désindustrialisation (- 4,3 millions de salariés). La baisse est en revanche plus contenue dans le cas des États-Unis (-28,7 \%, soit 3,3 millions de salariés), de l'Italie (-16\%) et de l'Espagne (- 9,1\%).

10 Tous les pays ayant connu la Révolution industrielle sont donc concernés à des degrés divers par ce phénomène, mais selon des modalités diverses (différences de spécialisation et de positionnement dans le bas, moyen et haut de gamme; compétitivité et productivité du travail plus ou moins grandes; marchés à l'export 
différents ; résiliences ou expositions très diverses des bassins d'emplois industriels à la désindustrialisation; etc.).

11 Quoiqu'il en soit la France et le Royaume-Uni apparaissent comme les pays qui se sont le plus désindustrialisés sur le plan de l'emploi au cours des dix dernières années au sein de l'UE. La rapidité et l'ampleur du phénomène y est même impressionnante, alors que leurs voisins sont eux aussi confrontés aux mêmes bouleversements liés aux effets conjugués de la mondialisation et de la crise économique mondiale (tableau 1). Inversement, des pays comme l'Allemagne (seconde puissance exportatrice au monde), le Japon ou la Suède ont su protéger, encourager, redéployer, voire sanctuariser des pans entiers de leur système productif. Des formes de réindustrialisation sont mêmes déjà à l'œuvre dans ces pays.

Tableau 1 : Évolution de l'emploi dans l'industrie manufacturière dans quelques grands pays développés (1991-2010, en \%)

\begin{tabular}{|l|l|}
\hline Pays & $\begin{array}{l}\text { Baisse de l'emploi en \% } \\
\text { (par ordre décroissant) }\end{array}$ \\
\hline Royaume-Uni & $-41,1 \%$ \\
\hline Japon & $-32,3 \%$ \\
\hline Allemagne & $-30,7 \%$ \\
\hline États-Unis & $-28,7 \%$ \\
\hline France & $-28,6 \%$ \\
\hline Suède & $-24,2 \%$ \\
\hline Corée du Sud & $-21,9 \%$ \\
\hline Italie & $-16,7 \%$ \\
\hline Espagne & $-9,1 \%$ \\
\hline
\end{tabular}

Source : Base de données AMECO, Commission européenne (cité par F. Bost, 2014)

La désindustrialisation des pays anciennement industrialisés a d'autant plus frappé les esprits ces dernières années que, concomitamment, la montée en puissance industrielle des pays émergents n'a eu de cesse de s'affirmer. La part des BRIC (Brésil, Russie, Inde et Chine) dans la production manufacturière mondiale est notamment passée de $11 \%$ à $27 \%$ au cours des dix dernières années. À cet égard, le fait que la Chine soit devenue en 2011 la première puissance manufacturière mondiale devant les États-Unis a fait office d'électrochoc, tout comme les États-Unis en leur temps lorsqu'ils avaient supplanté le Royaume-Uni à l'orée du XXe siècle. Aussi, la part des pays développés dans l'industrie manufacturière ne s'établit-elle plus qu'à $46 \%$ du total mondial, contre $60 \%$ dix ans plus tôt. Cette dynamique participe puissamment au mouvement de «basculement du monde " qui s'effectue au profit des pays émergents et, en premier lieu, de l'Asie (Carroué 2013), vers laquelle s'opère inexorablement le déplacement du centre de 
gravité de l'économie mondiale. Inquiétante pour les pays développés, qui se voient menacés dans leur prospérité, cette vision est cependant réjouissante pour les pays en développement concernés, qui n'y voient qu'un mouvement de rééquilibrage auxquels ils aspirent depuis longtemps.

Pour reprendre l'expression proposée dès 2005 par les économistes Lionel Fontagné et Jean-Hervé Lorenzi, la désindustrialisation contribuerait à la "perte de substance économique » des États ${ }^{1}$.

Les questions qui sous-tendent la réflexion sur ce sujet très géographique par ses implications territoriales fortes sont nombreuses et essentielles au débat sur l'avenir des pays européens. Au delà de l'instrumentalisation dont il fait souvent l'objet, à quoi renvoie exactement le phénomène de désindustrialisation? Celui-ci est-il incontrôlable, donc subi, entrainant inexorablement ces pays vers une rétrogradation dans le palmarès de l'économie mondiale? Ne serait-il pas plutôt inéluctable, mais susceptible d'être accompagné et anticipé dans le cadre d'une mutation globale des systèmes productifs de ces pays? Plus prosaïquement, les pays européens peuvent-ils espérer enrayer le phénomène et conserver des positions fortes en dépit du redéploiement partiel et irrépressible de pans entiers de leur industrie vers les pays émergents et les pays en développement dans le contexte de mondialisation contemporain? Des formes de réindustrialisation dans les territoires d'origine ne sontelles pas aussi pas envisageables (mais sous des modalités différentes), notamment en raison de la montée - inévitable à terme - des coûts de l'énergie et des contraintes de circuits plus courts en matière d'approvisionnement et de localisation des sites de production liés à la prise en compte des effets du réchauffement climatique?

Sur tous ces sujets fondamentaux, le débat reste encore largement ouvert. Mais le présent numéro de la Revue Géographique de l'Est montre que les spécialistes de géographie économique en Europe se sont bien réapproprié les questions industrielles, mais sous des angles renouvelés. La diversité des approches montre que le phénomène de désindustrialisation est bien devenu un objet géographique de premier ordre.

\section{Présentation du numéro}

Dans leur article intitulé «La désindustrialisation : quelles réalités dans le cas français ?", François Bost et Dalila Messaoudi se sont essentiellement focalisés sur les questions de la mesure et des origines du phénomène de désindustrialisation. Plus ancien qu'on ne le croit souvent, celui-ci est sans conteste l'un des plus vigoureux en Europe occidentale (-2,3 millions d'emplois perdus entre 1970 et 2014). L'originalité de cet article est de s'arrêter sur les principaux indicateurs statistiques de la désindustrialisation, de même que sur ses déterminants majeurs, qui en pondèrent la gravité : la hausse de la productivité du travail industriel (qui nécessite moins de maind'œuvre pour le même travail, ou même pour un travail plus important); l'externalisation des activités tertiaires - qui s'exerçaient jusque-là dans les entreprises industrielles - au profit de sociétés de services dont c'est la spécialité, ce qui provoque un glissement statistique qui n'a pas à inquiéter outre mesure ; enfin le manque de compétitivité des entreprises sous les effets de la « concurrence par les coûts », qui est par contre la cause la plus inquiétante de la désindustrialisation. Son second intérêt est de dresser un tableau cartographique de la désindustrialisation à l'échelle des 
304 zones d'emplois, approche qui fait généralement défaut dans les travaux et analyses portant sur la désindustrialisation en France.

À travers son article intitulé «La Lorraine industrielle de demain : quelques «citadelles »? Une désindustrialisation économiquement et géographiquement sélective" John Baude développe un exemple régional classique, mais qui méritait une mise au point très actualisée sur le sujet. Si, comme toutes les régions anciennement industrialisées, la Lorraine a perdu de nombreux emplois, la désindustrialisation s'y explique davantage par son manque global d'attractivité (cas notable des territoires vosgiens) et son plus faible niveau de qualification de la main-d'œuvre qui limitent les velléités d'investissement, notamment depuis l'étranger, que par sa trop forte spécialisation sectorielle. Au contraire, ce sont même des domaines de spécialité qui lui ont permis de contenir les pertes d'emplois (métallurgie, fabrication de produits métalliques, automobile). Les pistes de sortie de crise pour la région Lorraine sont désormais bien identifiées : innovation et réorientation affirmée vers des industries de conception.

Comment un pays aussi industrialisé que l'Allemagne a-t-il résisté aux crises successives et à la désindustrialisation? L'article de Michel Deshaies ( $D e$ la désindustrialisation à la ré-industrialisation : conséquences sur la géographie du territoire estallemand) apporte un éclairage original sur ce sujet, en insistant sur le rôle de la réunification des deux Allemagnes en 1990 dans la désindustrialisation de l'ex-RDA. Cette dernière a en effet connu une désindustrialisation massive - alors qu'elle était l'un des espaces les plus industrialisés au monde - qui s'est traduite par la perte en quelques années seulement des trois-quarts de ses emplois industriels. Face à ses conséquences économiques et sociales, le gouvernement fédéral a impulsé une dynamique puissante de réindustrialisation, en s'appuyant notamment sur un héritage d'activités particulièrement riche dans les Länder du sud (Saxe et Thuringe). Depuis 2000 , l'emploi industriel à l'est a ainsi progressé, tandis qu'il diminuait à l'ouest. Les nouveaux Länder de l'est ont ainsi retrouvé un niveau d'industrialisation supérieur à celui de la plupart des pays européens, ce qui constitue un cas tout à fait unique dans l'UE. Mais les contrastes géographiques demeurent marqués entre les noyaux industriels autour des grandes villes du sud, qui en ont profité et les anciens bassins miniers qui sont restés désindustrialisés. Cette nouvelle industrie, créée surtout par des investissements en provenance principalement de l'ouest de l'Allemagne, reste cependant très dépendante de décisions extérieures.

19 L'article de Henar Pascual et de Paz Benito (Territoires et paysages de la désindustrialisation en Espagne : des crises à la recherche d'opportunités) aborde la question de la désindustrialisation dans l'un des pays d'Europe du sud parmi les plus impactés. En effet, entre 1980 et 2013, la part de l'emploi industriel dans l'emploi total de l'Espagne a baissé de 27,2 à 13,6\%, tandis que la valeur ajoutée manufacturière passait de 24,4 à $12,2 \%$ dans le total de la valeur ajoutée. Le démantèlement du tissu productif a eu de fortes répercussions territoriales sur les régions espagnoles, surtout là où l'industrialisation était ancienne. Celles-ci sont alors entrées dans un processus de déclin, caractérisé par le désinvestissement, l'augmentation du chômage, la baisse de population et la crise urbaine. Les régions métropolitaines n'ont pas échappé à la restructuration industrielle. Cette dernière a altéré radicalement les caractéristiques de leur marché du travail, modifié leur base de production, leur tissu entrepreneurial et transformé leur physionomie du fait de la formation de friches industrielles. Cet article analyse les effets territoriaux de la désindustrialisation, recense les stratégies de 
revitalisation visant à inverser les processus de déclin (notamment les politiques régionales et locales) et identifie les principales actions impliquant un changement de paysage et de fonctionnalité dans des contextes urbains désindustrialisés.

Comment les pays d'Europe centrale et orientale ont-ils été frappés par la désindustrialisation? L'article d'Anna Geppert et de Iwona Pielesiak, intitulé Les transformations de l'industrie et leurs conséquences spatiales en Pologne (1989-2016) traite de ce sujet dans le cas de la Pologne, qui fut le plus grand des anciens pays satellites de l'ex-URSS. À l'instar d'autres pays de la sous-région, mais de manière sans doute plus marquée, la Pologne a connu un double mouvement de désindustrialisation / réindustrialisation, qui doit l'essentiel de sa dynamique à la dislocation du bloc de l'est, suite à la chute du communisme, de l'URSS et du CAEM (Conseil d'aide économique mutuelle, appelé aussi COMECON) à partir de 1990, et à son entrée rapide dans l'économie de marché et l'Union européenne. La perte des débouchés garantis par la division internationale du travail au sein du bloc socialiste a en effet provoqué l'effondrement des secteurs industriels les moins compétitifs et les plus vétustes, dont le coût social fut violent. Par contre, la Pologne a été l'un des pays de l'est qui a le plus bénéficié des flux d'IDE et de délocalisations d'activité industrielles en provenance d'Europe de l'ouest (depuis l'Allemagne en particulier), en raison certes de sa proximité géographique, mais surtout de sa main-d'œuvre qualifiée et peu chère. Aussi, plutôt que de désindustrialisation, c'est de transformation et de mutation de l'industrie qu'il convient de parler dans le cas polonais, dont les mutations ont été spectaculaires en à peine un quart de siècle. Si l'effondrement de l'industrie manufacturière traditionnelle et héritée de la période communiste n'a que rarement donné naissance à des modernisations réussies, en revanche, le développement de secteurs d'activités nouveaux et porteurs, de même que la recomposition des filières industrielles sont bien réels. Aujourd'hui, la Pologne est encore un pays largement industriel, où le secteur secondaire représente 30,2\% de l'emploi et 31,6\% du PIB. Certes l'emploi industriel a baissé (- 9,1\% depuis 1995), mais à un rythme trois fois moins rapide que la moyenne communautaire, tandis que la part de la valeur ajoutée brute industrielle a augmenté $(+8,2 \%)$, à contre-courant de la tendance européenne. Dans l'espace polonais, ces mutations produisent des effets différenciés qui accentuent les inégalités territoriales opposant l'ouest à l'est du pays, les métropoles aux villes petites et moyennes, les centres aux périphéries des villes. De nombreuses politiques de restructuration et de soutien aux activités industrielles ont été mises en œuvre. Le recul de près de trente ans depuis la chute du mur de Berlin permet d'interroger leur sens: s'agit-il de remèdes palliatifs, ou peuvent-elles réellement changer la donne?

21 L'analyse du cas d'école de l'espace métropolitain de Łódź, troisième ville de Pologne par son nombre d'habitants, par Monika Maria Cysek-Pawlak (Définir une stratégie de développement pour une ville post-industrielle. Analyse du cas de tódź en Pologne) complète l'approche précédente. L'auteur y montre comment l'ensemble des ressources territoriales y sont mobilisées selon plusieurs pistes de développement possibles, afin de réaliser différents projets de grande ampleur : infrastructures, reconversion du tissu urbain historique, etc. La question majeure concerne donc la cohérence des différentes stratégies adoptées. De plus, l'article souligne la nécessité de renforcer le rôle des investissements privés dans la nouvelle approche. 


\section{Sélection bibliographique francophone sur la désindustrialisation}

Ardinat Gilles, 2013, Géographie de la compétitivité, Paris, Puf, 214 p.

Artus Patrick, Virard Marie-Pierre, 2011, La France sans ses usines. Paris, Fayard, 171 p.

Artus Patrick, Virard Marie-Pierre, 2008, Globalisation. Le pire est à venir. Paris, La découverte, 154 p.

Asselain Jean-Charles, septembre 2012, Les caractéristiques de l'économie française : évolution depuis un demi-siècle, In Comprendre l'économie française, Problèmes économiques, La documentation Française, p. 5-15.

Askenazy Philippe, 28 mars 2012, Faut-il craindre la désindustrialisation ? Problèmes économiques, $\mathrm{n}^{\circ} 3040$, p. 35-40.

Benko Georges, 2008, La géographie économique : un siècle d'histoire, Annales de Géographie, 6, n 664, p. 23-49.

Benko Georges, Lipietz Alain, 2000, La richesse des régions. La nouvelle géographie socio-économique. Paris, PUF, $564 \mathrm{p}$.

Benko Georges, Lipietz Alain, 1992, Les régions qui gagnent, districts et réseaux. Les nouveaux paradigmes de la géographie économique, Paris PUF, coll. Économie en liberté, $424 \mathrm{p}$.

Bost François, 2013, Les pays développés à l'épreuve de la désindustrialisation. In Images économiques du monde 2014. Paris, Armand Colin, p. 9-26.

Bost François, 2012, Entreprises et territoires à l'épreuve de la mondialisation de l'économie. Université de Paris-Ouest-Nanterre-La Défense, Habilitation à diriger des recherches, 369 p.

Bost François. Territoires, entreprises et activités économiques à l'épreuve de la mondialisation, 2011, In Charvet Jean-Paul et Sivignon Michel. Géographie humaine, questions et lieux du monde contemporain. Paris, Armand Colin, p. 223-257.

Bost François, 2002, La localisation des activités économiques. In Charvet Jean-Paul et Sivignon Michel. Géographie humaine. Questions et enjeux du monde contemporain. Paris, Armand Colin, p. 221254.

Carroué Laurent, 2013, Crises et basculement des équilibres mondiaux. Images économiques du monde, Paris, Armand Colin, p. 8-27

Colletis Gabriel, 2012, L'urgence industrielle. Paris, Le bord de l'eau, 200 p.

DATAR, 2010, Territoires 2040. Aménager le changement. Paris, La documentation Française, 103 p. Url : http://territoires2040.datar.gouv.fr/IMG/pdf/territoires2040_n_1.pdf

DATAR, 2004, La France, puissance industrielle. Une nouvelle politique industrielle par les territoires. Paris, La Documentation française, $127 \mathrm{p}$.

Davezies Laurent, 2012, La crise qui vient. Paris, Le Seuil, 128 p. 
Davezies Laurent, 2010, La crise et nos territoires : premiers impacts. Rapport pour l'Assemblée des Communautés de France, la Caisse des Dépôts et l'Institut CDC pour la Recherche. Octobre, 96 p. Url : http://www.adcf.org/files/ADCF-etude-crise-20101105.pdf

Daviet Sylvie, 2005, Industrie, culture, territoire. Paris, L'Harmattan, 208 p.

Dicken Peter, 2003, Global Shift: Reshaping the Global Economic Map in the 21st Century (4 édition). London, Sage, $632 \mathrm{p}$.

Dunning John H., 2002, Regions, Globalization and the knowledge-based economy. Oxford, Oxford University Press, $506 \mathrm{p}$.

Dunning John H. Sarriana M. Lundan, 2002, Multinational enterprises and the Global Economy, (seconde édition). Northampton, Edward Elgar Publishing Limited, 891 p.

Fache Jacques, 2006, Les mutations industrielles. Paris, Belin, 190 p.

Fache Jacques, juillet 2006, Face à la mondialisation, la fin des certitudes de l'innovation et de la haute technologie. Historiens et géographes, ${ }^{\circ}$ 395, p. 167-170.

Fache Jacques, 2002, Les territoires de la haute technologie. Rennes, PUR, 157 p.

Fischer André, 1994, Industrie et espace géographique : introduction à la géographie industrielle. Paris, Masson,137 p.

Fischer André, Malézieux Jacques (dir.), 1999, Industrie et aménagement. Paris, L’Harmattan, 350 p.

Fontagné Lionel, Lorenzi Jean-Hervé, 2005, Désindustrialisation, délocalisations. Paris, La

documentation Française, 397 p.

Gallois Louis, 2013, Pacte pour la compétitivité de l'industrie française. Paris, La documentation Française, 2012, 67 p.

Hatem Fabrice, 2005, L'attractivité du territoire : de la théorie à la pratique. In Ministère de l'Economie et des Finances. L'industrie en France et la mondialisation. Paris, Service des Etudes et des Statistiques Industrielles, p. 269-283.

Holz Jean-Marc, Houssel Jean-Pierre, 2002, L'industrie dans la nouvelle économie mondiale. Paris, Puf, coll. Major, 449 p.

Le Blanc Gilles, mai 2012, Dynamiques des territoires industriels. Essai de prospective dans le cadre de « Territoires $2040 »$. Futuribles, n 385, p. 24

Le Blanc Gilles. Les espaces de la dynamique industrielle. In Territoires 2040. Paris DATAR, revue en ligne $n^{\circ}$ 3. Url : http://territoires2040.datar.gouv.fr/spip.php?article86\&revue=1

Le Blanc Gilles (dir), 2011, Des systèmes spatiaux en perspective. Territoires 2040. DATAR, 170 p. Url : http://territoires2040.datar.gouv.fr/IMG/pdf/territoires_2040_n_3.pdf

Leboutte René, 1977, Vie et mort des bassins industriels en Europe. 1750-2000. Paris, L'Harmattan, 592 p.

Mérenne-Schoumaker Bernadette, 2002, La localisation des industries. Enjeux et dynamiques. Rennes, Presses Universitaires de Rennes, $237 \mathrm{p}$.

Mérenne-Schoumaker Bernadette, 1998, Les délocalisations industrielles. Pour une clarification d'un phénomène mal connu et controversé. In G.Wakerman (Ed), Nouveaux espaces et systèmes urbains. Partis, SEDES, p. 257-267.

Montagné-Villette Solange, 2001, Le secondaire est-il soluble dans le tertiaire ? Annales de Géographie, $\mathrm{n}^{\circ}$ 110, vol. 617, p. 22-37. 
Morvan Yves (dir.), 2004, Activités économiques et territoires. Changement de décor. La Tour d'Aigues, Editions de l'Aube / DATAR, $176 \mathrm{p}$.

Morvan Yves, 1er trimestre 1983, La politique industrielle française depuis la Libération : quarante années d'interventions et d'ambiguïtés. Revue d'économie industrielle. Vol. 23, p. 19-35. Url : http://www.persee.fr/web/revues/home/prescript/article/ rei_0154-3229_1983_num_23_1_1138

Mouhoub El Mouhoud, 2006, Mondialisation et délocalisation des entreprises. Paris, La Découverte, Coll. Repères, $122 \mathrm{p}$.

Mucchielli Jean-Louis, 2008, La mondialisation. Chocs et mesure. Paris, Hachette supérieur, 159 p.

Nesta Lionel, 2010, Désindustrialisation ou mutation industrielle ? Economie et statistique, Vol. 438, n 438-440, p. 297-301. Url : http://www.persee.fr/web/revues/home/prescript/article/ estat_0336-1454_2010_num_438_1_9608

Pecqueur Bernard, janvier 2007, L'économie territoriale : une autre analyse de la globalisation. L'Economie politique, $\mathrm{n}^{\circ} 33$, p. 41-52.

Rainelli Michel, 1999, Les stratégies des entreprises face à la mondialisation. Paris, Editions EMS, 111 p.

\section{NOTES}

1. "La désindustrialisation est une tendance naturelle de nos économies, les délocalisations l'inévitable conséquence de la réorganisation globale des groupes; mais c'est la perte de substance industrielle, résultat de politiques inadaptées, qui menace notre niveau de vie » (p. 18).

\section{RÉSUMÉS}

Tous les pays européens, tant à l'ouest qu'à l'est, sont frappés par un phénomène de désindustrialisation. Ses principales conséquences sont la baisse sensible de l'emploi dans le secteur de l'industrie et la fragilisation des économies nationales et des territoires concernés. La crise économique des années 2007-2008 a révélé l'ampleur du phénomène et suscité une prise de conscience générale de l'importance de cette activité dans les stratégies de développement et de puissance des Etats. Mais la désindustrialisation revêt des aspects différents selon les Etats qui s'attèlent désormais à l'enrayer par différents moyens. Ce numéro de la Revue géographique de l'est analyse ce phénomène de la désindustrialisation à partir de quelques exemples de pays européens choisis pour leur représentativité (France, Allemagne, Pologne, Espagne), ou de cas d'école régionaux (Lorraine) ou urbain (Lodz en Pologne).

Western and Eastern European countries alike are affected by deindustrialization. Its main consequences are a significant decline in industrial employment and the weakening of national and local economies. The 2007-2008 economic crisis revealed the extent of this phenomenon and led to an increased awareness of the major part played by industrial activities in the states' strategies to develop and to gain and retain power. However, deindustrialization takes on different forms in the states that are now trying to stop it through different means. This issue of 
the Revue géographique de l'est examines this deindustrialization process using a few European states as representative examples (France, Germany, Poland, Spain) as well as regional (Lorraine) or urban (Lodz in Poland) textbook cases.

Alle europäischen Länder, sowohl im Westen als auch im Osten, sind von einem Phänomen der Entindustrialisierung betroffen. Ihre wichtigsten Konsequenzen sind der deutliche Rückgang der Beschäftigung im Industriesektor und die Schwächung der nationalen Volkswirtschaften und der betroffenen Gebiete. Die Wirtschaftskrise der Jahre 2007-2008 hat das Ausmaß des Phänomens aufgezeigt und ein allgemeines Bewusstsein für die Bedeutung dieser Aktivität in den Entwicklungs- und Machtstrategien der Staaten geweckt. Die Entindustrialisierung hat jedoch unterschiedliche Aspekte, die von den Staaten abhängen, die jetzt versuchen, sie mit verschiedenen Mitteln zu stoppen. Diese Ausgabe der geographischen Zeitschrift von Osten analysiert dieses Phänomen der Deindustrialisierung anhand einiger Beispiele europäischer Länder, die aufgrund ihrer Repräsentativität (Frankreich, Deutschland, Polen, Spanien) oder regionaler (Lothringen) oder städtischer Fallstudien ausgewählt wurden (Lodz in Polen).

\section{INDEX}

Mots-clés : mondialisation, désindustrialisation, réindustrialisation, industries, entreprises

Keywords : globalization, deindustrialization, reindustrialization, industry, firms

Schlüsselwörter : Globalisierung, Deindustrialisierung, Reindustrialisierung, Industrie, Unternehmen

\section{AUTEUR}

\section{FRANÇOIS BOST}

Professeur des universités, Université de Reims Champagne-Ardenne (URCA), 57 rue Pierre Taittinger, 51096 REIMS Cedex <francois.bost@univ-reims.fr> 